Document downloaded from:

http://hdl.handle.net/10251/44373

This paper must be cited as:

Valero-Nogueira, A.; Herranz Herruzo, Jl.; Baquero Escudero, M.; Hernández-Murcia, R.; Rodrigo Peñarrocha, VM. (2013). Practical derivation of slot equivalent admittance in periodic waveguides. IEEE Transactions on Antennas and Propagation. 61(4):2321-2324. doi:10.1109/TAP.2012.2231934.

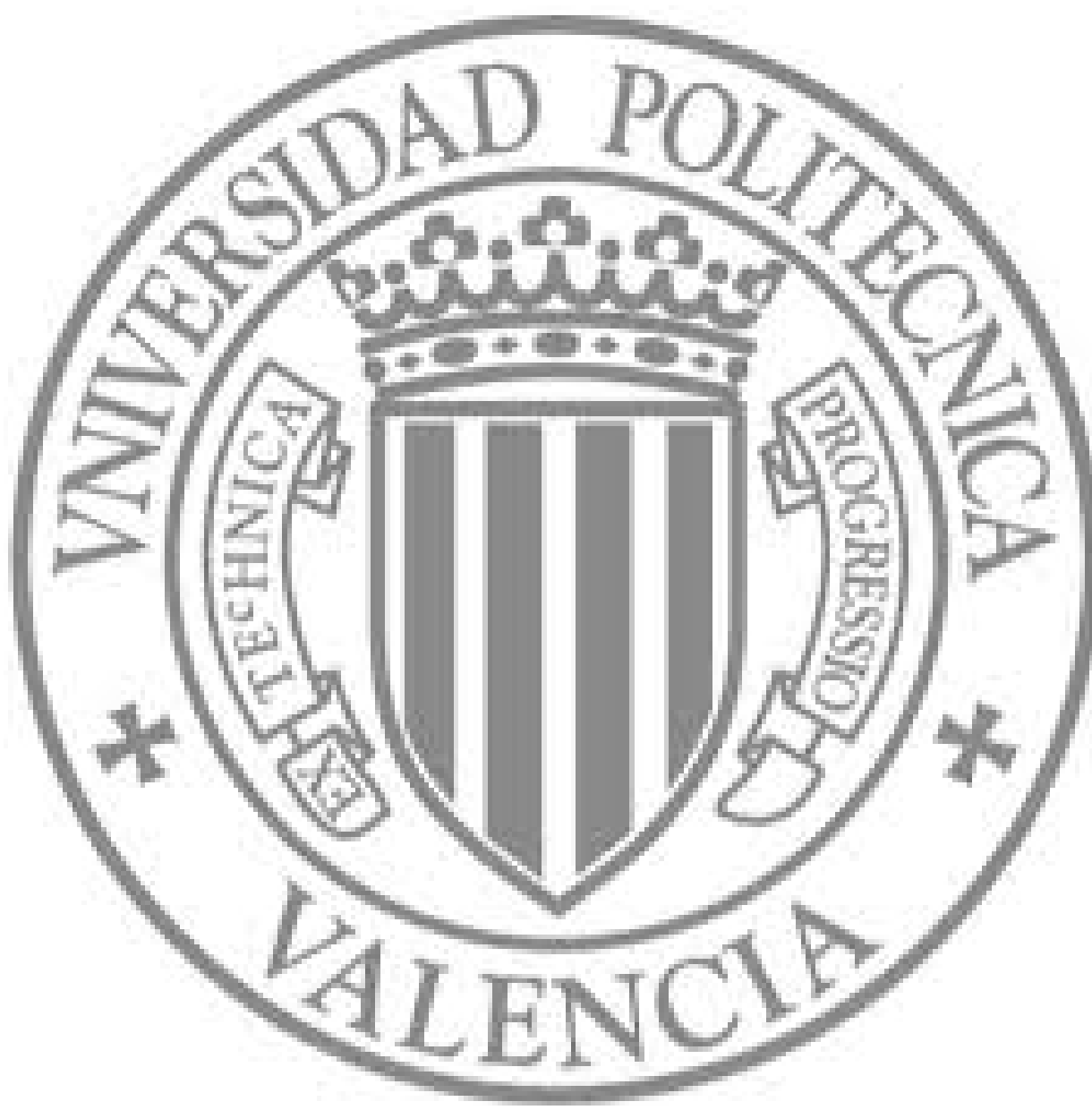

The final publication is available at

http://dx.doi.org/10.1109/TAP.2012.2231934

Copyright Institute of Electrical and Electronics Engineers (IEEE) 


\title{
Practical Derivation of Slot Equivalent Admittance in Periodic Waveguides
}

\author{
Alejandro Valero-Nogueira, IEEE Senior Member, Jose I. Herranz-Herruzo, IEEE Member, Mariano Baquero, \\ IEEE Member, Rafael Hernández-Murcia, Vicent Rodrigo
}

\begin{abstract}
This paper describes a procedure to obtain the equivalent-admittance representation of a slot in a waveguide. The approach is general but it is particularly interesting for periodic waveguides because it does not require to know the waveguide's Green's function nor to properly define the ports in such waveguides. The procedure is demonstrated on a wellknown rectangular waveguide. It is later applied to a periodic waveguide and it is used to design a small array. Measurements of the array performance are shown to validate the approach.
\end{abstract}

Index Terms-Corrugated waveguides, gap waveguides, periodic structures, slot antennas, slot arrays

\section{INTRODUCTION}

$\mathbf{P}$ ERIODIC waveguiding structures have been used for decades for a variety of applications in microwave, millimeter-wave, and optic-wave regions [1]. More recently these structures have gained renewed interest thanks to the development of novel artificial surfaces and the introduction of composite right/left handed waveguides [2]. Also, newly introduced contactless waveguides capable of confined wave propagation, resort to the high impedance effect of a periodic bed of nails to avoid contact between metal plates [3]. The analysis of periodic transmission lines is usually performed by invoking Bloch-Floquet theorem in order to determine the propagation constant of the supported wave.

When a nonperiodic obstacle is present in the periodic waveguide, in the form of a slot, an iris, a coaxial probe, etc, the direct use of Floquet's theorem is not applicable since it assumes there is a fixed progressive phase shift from one unit cell to the other, which is not the case when an obstacle is there. The theoretical modeling of a periodic structure with an aperiodic obstacle in it, or with a localized source is a challenging problem addressed already in the past in [4] among others. Given the complexity of the problem, most analyses up to date focus on very specific structures. The modeling of arbitrary obstacles in periodic structures is still an open problem even from a numerical point of view. In this regard, an accurate procedure to indirectly obtain S-parameters using data obtained from commercial electromagnetic simulators was recently published [5].

The kind of obstacle considered in this work is a rectangular shunt slot. Nonetheless, the approach is in fact valid for any type of aperture. Determination of the Equivalent-Admittance

A. Valero-Nogueira, J.I. Herranz-Herruzo, M. Baquero and R. HernándezMurcia and Vicent Rodrigo, are with Universidad Politécnica de Valencia, 46022 Valencia, Spain (email: avalero@dcom.upv.es). This work was supported by the Spanish Ministerio de Ciencia e Innovación and the European Regional Funds, under project TEC2010-20841-C04-01 and CSD2008-00068 representation of shunt slots is a necessary first step before being able to design a slotted waveguide array. In the usual procedure, $Y_{\text {shunt }}$ is obtained from the $S_{11}$-parameter of a slot alone using $Y_{\text {shunt }}=-2 S_{11} /\left(1+S_{11}\right)$.

When dealing with periodic waveguides the Green's function of a current in it is not known to be able to calculate $S_{11}$ from a Method of Moments approach. Furthermore, if a purely numerical approach is going to be followed using commercial electromagnetic suites, port definition is limited to continuous cross-section waveguides. These ports, if used, would introduce a discontinuity between the periodic waveguide and the port itself, leading to deceiving results in the $Y_{\text {shunt }}$ calculation. This is illustrated in Fig. 1.

In this paper an indirect approach is proposed to obtain the $S_{11}$-parameter associated to a slot in a waveguide. In brief, the procedure requires the evaluation of an excitation field within the periodic waveguide in the absence of the slot and the $S_{22^{-}}$ parameter calculated using the slot as if it were an input port (See Fig. 1).

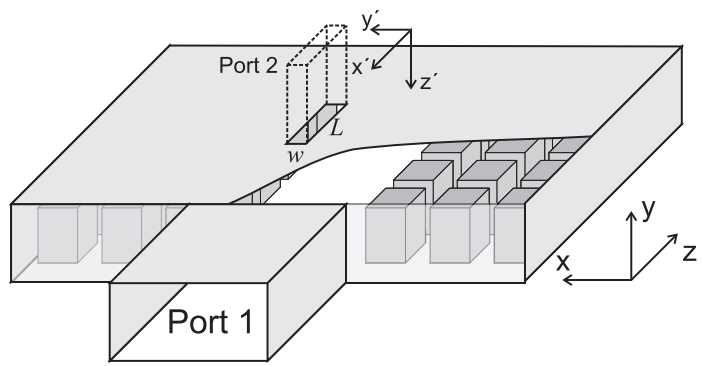

Figure 1. Waveguide exhibiting a periodic profile with a longitudinal slot in the upper plate

\section{Reflection CoefFicient DeRIVATION}

The indirect derivation of the reflection coefficient is performed starting from an aperture integral formulation applied to the slot. The derivation can also be performed from basic microwave network analysis as in [6], but the current approach is much more straightforward.

\section{A. Aperture Integral Equation}

The aperture integral equation (AIE) is the standard procedure to deal with apertures on conducting surfaces. Let us assume the aperture is a slot for simplicity. The AIE essentially establishes an integral equation by formulating two equivalent problems for the two regions, let us name them $a$ and $b$, 
connected through the aperture. These two problems become independent as the slot is closed by a conducting surface. Continuity of tangential electric fields in the aperture is assured by imposing opposite equivalent magnetic currents on both sides of the closed aperture, while the enforcement of the continuity condition of tangential magnetic fields yields the operator equation,

$$
\mathbf{H}_{\text {tan }}^{a}(\mathbf{M})=\mathbf{H}_{\text {tan }}^{b}(-\mathbf{M})+\mathbf{H}_{\text {tan }}^{i n c}
$$

whose solution determines the unknown equivalent magnetic current $M$.

An approximate solution for $\mathbf{M}$ in (1) can be obtained using the Method of Moments (MoM). Let us consider a slot of length $L$ and width $w$. Let $\left\{\frac{1}{w} \mathbf{m}_{n}\right\}$ be a set of $\mathbf{N}$ basis functions such that $\mathbf{M}=\sum \frac{v_{n}}{w} \mathbf{m}_{n}$ and $\left\{\mathbf{w}_{n}=\frac{1}{w} \mathbf{m}_{n}\right\}$ a set of weighting functions. For convenience, the basis and weighting functions are of the sinusoidal type in the slot's long dimension and constant in the narrow one: $\mathbf{m}_{n}=\sin \left(\frac{n \pi}{L} x^{\prime}\right) \hat{\mathbf{x}}^{\prime}$. Therefore, the integral equation can be written in matrix form as

$$
\left[Y^{a}+Y^{b}\right][V]=[I]
$$

where $\left[Y^{a}\right]=\left[\left\langle\mathbf{w}_{m}, \mathbf{H}_{t a n}^{a}\left(\mathbf{m}_{n}\right)\right\rangle\right]_{N \times N}$ is the external admittance matrix, $\left[Y^{b}\right]=\left[\left\langle\mathbf{w}_{m}, \mathbf{H}_{\text {tan }}^{b}\left(\mathbf{m}_{n}\right)\right\rangle\right]_{N \times N}$ the internal one and $[I]=\left[\left\langle\mathbf{w}_{m}, \mathbf{H}_{t a n}^{i n c}\right\rangle\right]_{N \times 1}$ the excitation vector. As shown in (2), the aperture admittance is the parallel of two admittances which correspond to two independent situations seen from the aperture, as emphasized above.

The external problem is usually approximated by an infinite ground plane. Therefore $Y^{a}$ is obtained applying image theory and the free-space Green's function. On the other hand the internal admittance calculation would require the knowledge of the Green's function of the waveguide at hand, which is known only for a bunch of cases. Alternatively, the internal admittance can be regarded as the loading admittance of a rectangular waveguide showing the slot's cross-section, as sketched in Fig. 1. This waveguide presents a multimode reflection coefficient, $S_{22}$, associated to the first $\mathrm{N} T E_{n 0}$ modes of interest, which can be computed numerically. The transverse electric field of these modes behave like the basis functions chosen above. Then, from basic network theory, the normalized internal admittance can be calculated as

$$
\left[\overline{Y^{b}}\right]=\left[I+S_{22}\right]^{-1}\left[I-S_{22}\right]
$$

where $[I]$ is the identity matrix.

It is noteworthy to observe at this point that the main waveguide ends must be properly matched for the $S_{22}$ coefficient to provide the internal admittance correctly since the waveguide is supposed to be infinite for the $Y_{\text {shunt }}$ calculation. This important aspect will be discussed in more detail in next section. For those cases in which slot thickness must be taken into account, either it can be included in the numerical model as part of the internal admittance or modeled separately with a two-port generalized admittance matrix and combined with the external or the internal admittance.
Vector $[I]$ in (2) is normally calculated using the fundamental mode of the waveguide as the excitation field. For the purpose of this derivation it is useful to assume that this field is normalized to have unit power and it is originated by a known magnetic current $\mathbf{M}_{0}$, such that $\mathbf{M}_{0}=\mathbf{E}_{0} \times \mathbf{n}$, being $\mathbf{E}_{0}$ the transverse electric field component of the waveguide's fundamental mode, and $\mathbf{n}$ a unit vector oriented in the longitudinal direction of the waveguide. Once the excitation vector is known, equation (2) is solved for the voltage vector.

\section{B. Reflection Coefficient}

In our case, the reflection coefficient sought is the ratio of the field scattered back from the slot to the incident field radiated by $\mathbf{M}_{0}$. Any field component can be chosen to establish this ratio. Here it is convenient to choose the transverse magnetic field. With all the assumptions made, the $S_{11}$-parameter can now be written as

$$
S_{11}=\frac{H_{t}^{\text {scattered }}}{H_{t}^{\text {inc }}}=\left\langle\mathbf{M}_{0}, \mathbf{H}_{t}^{\text {scattered }}(\mathbf{M})\right\rangle
$$

where $\mathbf{M}$ is the magnetic current known after solving (2).

The difficulty at this point, however, is that since the waveguide's Green's function is not known, it is not possible to obtain the scattered field directly. Yet, since we are not interested in the scattered field itself but in the ratio of the scattered to the incident field, the reciprocity theorem can be invoked to state that

$$
\left\langle\mathbf{M}_{0}, \mathbf{H}_{t}^{\text {scattered }}(\mathbf{M})\right\rangle=\left\langle\mathbf{M}, \mathbf{H}_{\text {tan }}^{\text {inc }}\left(\mathbf{M}_{0}\right)\right\rangle
$$

which can be written very nicely in matrix form with the help of (2) as

$$
S_{11}=[I]^{t}[V]=[I]^{t}\left[Y^{a}+Y^{b}\right]^{-1}[I]
$$

where the superscript $t$ means transpose.

After this derivation it is evident that (6) is in fact valid for any aperture size. Furthermore, it can be used for arbitrary cross-section waveguides both continuous and periodic ones. It can also be extracted that the expression is formally exact, being its accuracy bounded by the intrinsic precision of the numerical method used to compute $\left[Y^{b}\right]$.

\section{RESULTS}

A series of examples will be considered below in order to show the accuracy achievable and the practical implementation of expression (6).

\section{A. Shunt Slot in Rectangular Waveguide}

Initially, a well-documented case such as a shunt slot in a rectangular waveguide will be considered for reference purposes. The $S_{11}$-parameter can be obtained using different techniques. Here the reflection coefficient will be calculated using an accurate custom MoM code which uses the waveguide Green's function. In addition, results obtained using CST's frequency domain solver [7] will be shown. We choose in particular a WR-90 waveguide and select one of the cases 
treated by Stern and Elliott in [8], where a very detailed study can be found.

In this case the slot thickness is zero. The internal admittance in (6) is calculated placing a waveguide port at the slot and computing the generalized scattering parameters of five excitation modes while the waveguide is terminated with waveguide ports, i.e, it is perfectly matched. This computation is performed with CST. The external admittance is computed with the custom MoM code.

As explained above, each term of the excitation vector is calculated from

$$
I_{n}=\frac{1}{\sqrt{2 P_{0}}} \iint_{\text {slot }} \sin \left(\frac{n \pi}{L} x^{\prime}\right) H_{x}^{i n c} d S_{\text {slot }}
$$

$H_{x}^{i n c}$ is computed numerically at the interface between internal and external admittances using CST again but feeding the waveguide from one end this time, and in the absence of any port or physical aperture at the slot location. Magnetic field amplitude must be normalized with respect to the input power which in general will be $P_{0}=1-\left|S_{11}\right|^{2}$. Besides, incident field phase requires to be referenced to the slot's center for the $Y_{\text {shunt }}$ calculation.

Fig. 2 shows the slot's equivalent admittance calculated at 9 $\mathrm{GHz}$ using (6) and the other two procedures mentioned above. The figure also shows a black dot which indicates the resonant length obtained by Stern and Elliott for this case (slot offset of $6.35 \mathrm{~mm}$ ).

The most accurate results are those obtained with the Method of Moments. The number of basis functions was increased up to a convergence point. This happened for 18 basis functions and the resonant length appeared to be very close to Stern and Elliott's. The indirect method proposed here is shown in black line. These results were obtained for 5 modes. Simulations using more modes were also performed but very poor convergence was observed. If fact with only one mode, results were almost the same as those in the figure. The reason for this lack of convergence is attributed to numerical inaccuracies in the computation of the generalized scattering matrix. When the slot is shorter than half wavelength, all modes considered in the computation are in fact evanescent. This behavior increases with the order of the mode up to a point in which numerical errors may exceed the actual mode weight. We believe that other type of numerical techniques such as Mode Matching may produce better results.

\section{B. Shunt Slot in a Waveguide With Dielectrically Filled Cor- rugations}

The situation considered now is a longitudinal slot in a waveguide with transverse corrugations filled with a dielectric material. This problem was studied previously with another indirect method described in [5]. This is one of the few, if not the only, periodic case for which the $S_{11}$-parameter of a slot is available for reference.

As in III-A, the first step is matching the waveguide ends so that reflections are suppressed or at least minimized. For that purpose the matched transitions designed in [5] are used.

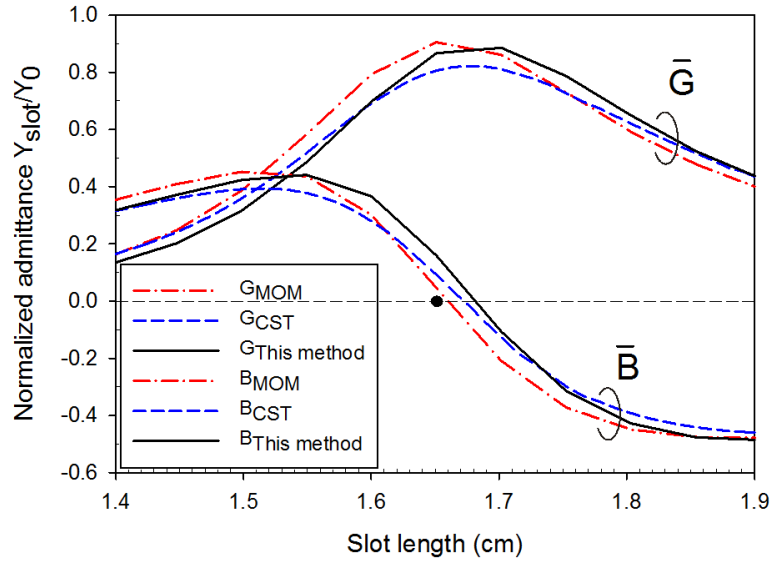

Figure 2. $Y_{\text {shunt }}$ of a longitudinal slot in a WR-90 waveguide calculated at $9 \mathrm{GHz}$ with three different methods. The slot's offset is $6.35 \mathrm{~mm}(0.25$ inches) and width is $1.6 \mathrm{~mm}$. Slot is considered of zero thickness, except for the CST model where a thickness of $t=0.05 \mathrm{~mm}$ was used to enhance numerical accuracy. The black dot in the middle indicates the resonant length obtained by Stern and Elliott in [8] for this case.

The geometry and all representative dimensions are shown in Fig. 3. Besides the corrugated waveguide, two stepped transitions to WR-90 can be seen on both waveguide ends. The relative permittivity of the grooves' filling is $\varepsilon_{r}=2.17$. Slot offset is $\delta_{y}=1.5 \mathrm{~mm}$.

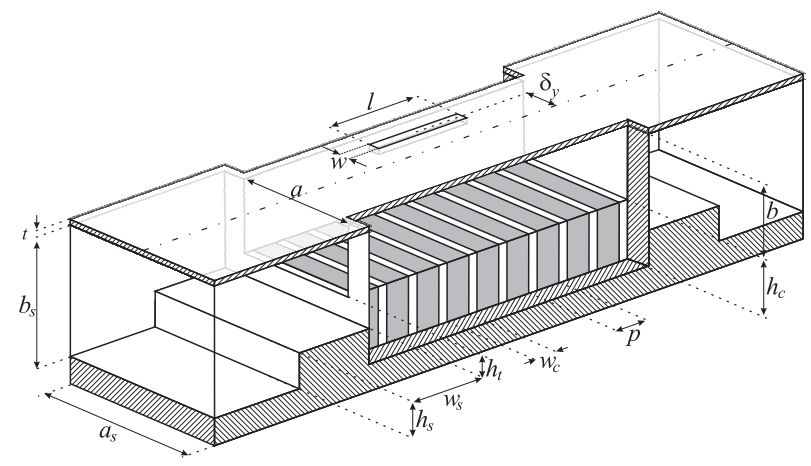

Figure 3. Corrugated waveguide with input and output stepped transitions to WR-90. Side walls have been removed to show the waveguide. Dimensions for the corrugated waveguide are $a=16 \mathrm{~mm}, b=4 \mathrm{~mm}, h_{c}=12.6 \mathrm{~mm}$, $p=3.21 \mathrm{~mm}$, and $w_{c}=3.175 \mathrm{~mm}$. Dimensions for the stepped transitions are $a_{s}=22.86 \mathrm{~mm}, b_{s}=10.1 \mathrm{~mm}, h_{s}=2.45 \mathrm{~mm}, h_{t}=3.71$ and $w_{s}=8.6 \mathrm{~mm}$. Slot width is $w=1.0 \mathrm{~mm}$, thickness $t=0.45 \mathrm{~mm}$ and offset $\delta_{y}=1.5 \mathrm{~mm}$.

Fig. 4 shows the $Y_{\text {shunt }}$ for structure in Fig. 3 as a function of slot's length. The working frequency is now $8.5 \mathrm{GHz}$. Agreement with respect to the reference method is very remarkable, particularly the resonant length and the conductance amplitude, which are the two quantities sought out in slotted waveguide array design. As in III-A, five modes were used in this analysis too.

\section{ARRAY DESIGN}

In this last section we apply the new formula to the design of a small array in a periodic waveguide. The waveguide chosen is a groove gap waveguide [9], a new type of waveguide 


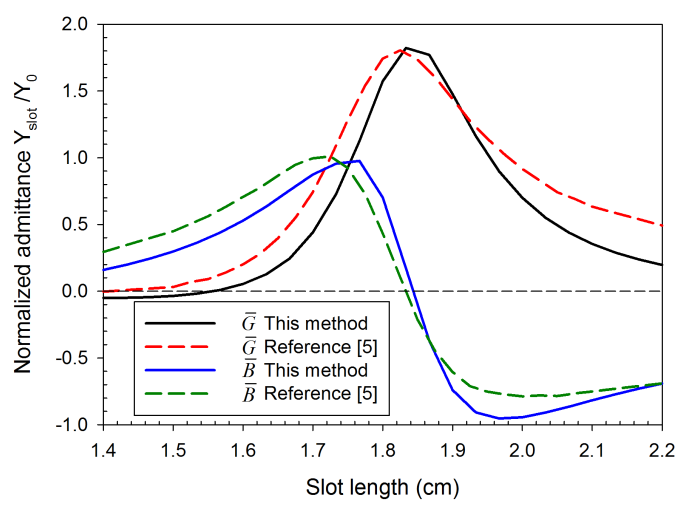

Figure 4. Normalized equivalent shunt admittance of a longitudinal slot in the waveguide shown in Fig. 3 at $f=8.5 \mathrm{GHz}$, as a function of slot length. Offset, $\delta_{y}=1.5 \mathrm{~mm}$, slot width, $w=1.0 \mathrm{~mm}$. Simulation using proposed method is compared with data provided in [5].

which does not need metal contact between upper and lower conductor. The waveguide consists of a textured surface for the lower conductor and a conducting plate for the upper one. The textured surface is a 2D periodic bed of nails, which are a quarter-wavelength in height, that creates a high impedance condition in the gap between the nails top and the upper plate. Such condition suppresses any propagation in the gap but if a set of nails is removed to open a groove, propagation can take place there. Fig. 5 shows the textured surface. The bed of nails shown has been designed to operate from 25 to $50 \mathrm{GHz}$. Relevant dimensions are $w_{n}=1.2 \mathrm{~mm}, h_{n}=2 \mathrm{~mm}$ and $p=3$ $\mathrm{mm}$ for width, height and periodicity of nails, respectively. The gap between nails top and upper plate is $d=0.4 \mathrm{~mm}$.

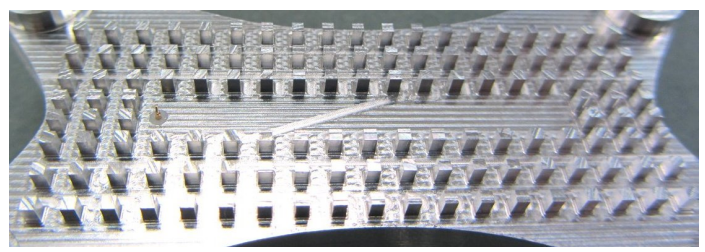

Figure 5. Groove gap waveguide. Nails width, height and periodicity are $1.2 \mathrm{~mm}, 2 \mathrm{~mm}$ and $3 \mathrm{~mm}$, respectively. Groove width and length are $5.4 \mathrm{~mm}$ and $38 \mathrm{~mm}$, respectively.

The groove waveguide can propagate a fundamental mode very similar to the $T E_{10}$ mode of a conventional rectangular waveguide. To that purpose, waveguide dimensions have been chosen to be $a=5.4 \mathrm{~mm}, b=2.4 \mathrm{~mm}$ and $L=38$ $\mathrm{mm}$ for width, height and length, respectively. The array of shunt slots is of the resonant type, being $37.5 \mathrm{GHz}$ the design frequency and $N=5$ the number of elements. The amplitude taper chosen is an ordinary triangular distribution. The array is designed following the conventional procedure described in [10]. The waveguide was fed with a coaxial probe.

In Fig. 6, the H-plane pattern is shown. The correspondence between measured and simulated main lobe for the H-plane is very good.

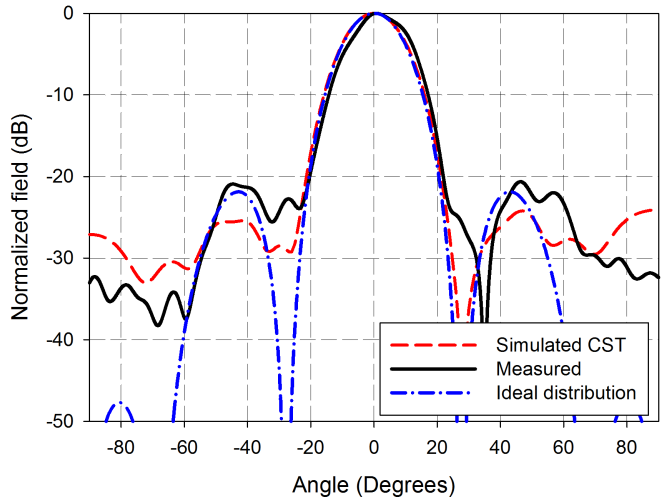

Figure 6. H-plane pattern of slot array with triangular distribution

\section{CONCLUSIONS}

A useful procedure to determine the equivalent admittance of a slot in a periodic waveguide has been described, evaluated and verified experimentally. Expression (6) is theoretically exact and in fact can be used not only for periodic waveguides but also for continuous waveguides of arbitrary shape, or waveguides having arbitrary obstacles within. The inaccuracies detected during the computations are due to the numerical procedure used for the computation of the reflection coefficient at the slot, when it is seen as a port, due to the evanescent nature of the modes involved.

\section{ACKNOWLEDGMENTS}

The authors express their gratitude to Professor Jaime Esteban from Universidad Politécnica de Madrid, Spain, for providing the reference data used in Fig. 4.

\section{REFERENCES}

[1] C. Elachi, "Waves in active and passive periodic structure: A review," Proceedings of the IEEE, vol. 64, no. 12, pp. 1666-1668, 1976.

[2] I. Eshrah, A. Kishk, A. Yakovlev, and A. Glisson, "Rectangular waveguide with dielectric-filled corrugations supporting backward waves," IEEE Trans. on Microwave Theory and Techniques, vol. 53, no. 11, pp. 3298 - 3304, Nov. 2005.

[3] P.-S. Kildal, E. Alfonso, A. Valero-Nogueira, and E. Rajo-Iglesias, "Local metamaterial-based waveguides in gaps between parallel metal plates," IEEE Antennas and Wireless Propagation Letters, vol. 8, pp. 84 $-87,2009$.

[4] G. Valerio, P. Burghignoli, P. Baccarelli, and A. Galli, "Input impedance of nonperiodic sources exciting 1-d periodic shielded microstrip structures," IEEE Trans. on Microwave Theory and Techniques, vol. 58, no. 7, pp. $1796-1806$, July 2010.

[5] M. Navarro-Tapia, J. Esteban, J. Varela, and C. Camacho-Penalosa, "Simulation and measurement of the S-parameters of obstacles in periodic waveguides," IEEE Trans. on Microwave Theory and Techniques, vol. 60, no. 4, pp. 1146 -1155, April 2012.

[6] J. Herranz-Herruzo, A. Valero-Nogueira, M. Baquero-Escudero, R. Hernández-Murcia, "Equivalent-admittance representation in periodic waveguides," Antennas and propagat. Symp. Chicago, 2012.

[7] CST Microwave Studio 2012. Available at: www.cst.com.

[8] G.J. Stern, R.S. Elliott, "Resonant length of longitudinal slots and validity of circuit representation: theory and experiment," IEEE Trans. on Antennas and Propagation, vo. 33, no. 11, pp. 1264-1271. Nov. 1985.

[9] E. Rajo-Iglesias, P.-S. Kildal, "Groove gap waveguide: A rectangular waveguide between contactless metal plates enabled by parallel-plate cut-off," European conf. on antennas and propagat., EuCAP. 2010.

[10] R.C. Johnson, Antenna engineering handbook, 3rd edition, chap. 9, pp. 20-22, McGraw-Hill, 1993. 